DOI: $10.2478 / \mathrm{v} 10014-011-0023-7$

COBISS Code 1.01

Agrovoc descriptors: wines, volatile compounds, flavour, flavour compounds, aroma precursors, analytical methods

Agris category code: Q04

\title{
Validation of the method for the determination of some wine volatile compounds
}

\author{
Dejan BAVČAR ${ }^{1}$, Helena BAŠA ČESNIK ${ }^{1}$
}

Received: July 26, 2011; accepted: September 19, 2011.

Delo je prispelo 26. julija 2011, sprejeto 19. septembra 2011.

\begin{abstract}
Wine aroma is influenced by a number of volatile compounds. This article describes the validation of the method for 26 volatile compounds found in wine. Volatile compounds were determined with discontinuous liquid-liquid extraction and GC-MS detection. It was determined, that the method is linear with square correlation coefficient ranging from 0.961 to 0.999. Limits of quantitative determination range from 0.52 $\mu \mathrm{g} / \mathrm{L}$ to $14.8 \mu \mathrm{g} / \mathrm{L}$. Recoveries range from $71.1 \%$ to $105.7 \%$ except for two compounds with lower recoveries. Measurement uncertainty ranges from $5.0 \%$ to $28.9 \%$. According to the validation, the method is suitable for the determination of at least 24 volatile compounds common to wine. A practical method application was presented on Zelen wine variety from two different production procedures.
\end{abstract}

Key words: wine, aroma, volatile compounds, GC-MS

\author{
IZVLEČEK \\ VALIDACIJA METODE ZA DOLOČANJE \\ NEKATERIH HLAPNIH SPOJIN V VINU
}

$\mathrm{Na}$ aromo vina vplivajo številne hlapne spojine. Ta članek opisuje validacijo metode za 26 hlapnih spojin, ki jih najdemo $\mathrm{v}$ vinu. Hlapne spojine so bile določene $\mathrm{z}$ diskontinuirano ekstrakcijo tekoče-tekoče in GC-MS detekcijo. Določili smo, da je metoda linearna, $\mathrm{z}$ razponom kvadrata korelacijskega koeficienta od 0,961 do 0,999. Meje kvantitativne določitve imajo razpon od $0,52 \mu \mathrm{g} / \mathrm{L}$ do $14,8 \mu \mathrm{g} / \mathrm{L}$. Izkoristki imajo razpon od $71,1 \%$ do $105,7 \%$, razen za dve spojini, katerih izkoristek je nižji. Merilna negotovost ima razpon od 5,0\% do $28,9 \%$. Z ozirom na validacijo lahko potrdimo primernost metode za določanje vsaj 24 hlapnih spojin značilnih za vino. Praktični prikaz uporabe metode smo predstavili na vinih sorte Zelen iz dveh različnih postopkov pridelave.

Ključne besede: vino, aroma, hlapne spojine, GC-MS

Prispevek je del doktorske disertacije z naslovom "Vpliv maceracije na aromatične značilnosti primorskih belih vin", mentorica prof. dr. Tatjana Košmerl

\section{INTRODUCTION}

Wine aroma, a very important sensory parameter is produced by a complex balance of several volatiles. More than 800 volatile compounds such as alcohols, esters, phenols, monoterpenes, norisoprenoides, lactones, aldehydes and ketones have been identified in wine (Selli et al., 2004; Tamborra et al., 2004).

The wine aroma is complex due to a large number of compounds present and their different chemical nature with a wide range of polarity, volatility, solubility and $\mathrm{pH}$ values. Therefore the sample preparation and particularly the extraction and concentration of volatile compound are an important factor in their determination (Cabredo Pinillos et al., 2004).

Appropriate extraction of wine volatile compounds must be performed before their detection. Exceptionally so called major wine volatile compounds present in $\mathrm{mg} / \mathrm{l}$, like acetaldehyde, ethyl acetate, methanol and higher alcohols are detected directly without previous extraction where samples are only diluted and deacidificated prior to analysis (Peinado et al., 2004; Lukić et al., 2008).

\footnotetext{
1 Agricultural Institute of Slovenia, Hacquetova ulica 17, SI-1000 Ljubljana, Slovenia, Ph.D., e-mail: dejan.bavcar@kis.si, helena.basa@kis.si
} 
Extraction of minor volatile compounds, present in wine in $\mu \mathrm{g} / \mathrm{L}$, is done today mostly in three different ways. The first is discontinuous or continuous liquid-liquid extraction (LLE) of wine with organic solvent. Both discontinuous and continuous liquid-liquid extractions are suitable to measure volatiles, but to perform a second one special apparatus must be provided and main disadvantages, like time consuming process and large volumes of solvents, are not avoided (Cabredo Pinillos et al., 2004). As solvents mainly dichloromethane (Selli et al., 2003) or mixture of pentane: dichloromethane $=60: 40$ (Pérez-Coello et al., 2003; Izquierdo et al., 2008) are used. The second approach is solid phase extraction (SPE) using Sep Pack $\mathrm{C}_{18}$ cartridges (Tamborra et al., 2004) or LiChrolut EN resins (Loscos et al., 2010; Sáenz-Navajas et al., 2010). The third approach is Solid Phase Micro Extraction (SPME) with different fibers used: carbowaxdivinylbenzene (Lambropoulos and Roussis et al., 2007; Antalick et al., 2010), polydimethylsiloxane (Nasi et al., 2008; Antalick et al., 2010), polydimethylsiloxane/ divinylbenzene (Nasi et al., 2008; Antalick et al., 2010), carboxen/polydimethylsiloxane (Nasi et al., 2008; Antalick et al., 2010) or divinylbenzene/carboxen/ polydimethylsiloxane (Nasi et al., 2008; Antalick et al., 2010).

Detection of volatile compounds is performed by gas chromatograph (GC) coupled with Flame Ionisation Detector (FID) (Pérez-Coello et al., 2003; Selli et al., 2003; Selli et al., 2004; Tamborra et al., 2004; Selli et al., 2006; Loscos et al., 2010) or mass spectrometer (MS) (Pérez-Coello et al., 2003; Selli et al., 2003; Selli et al., 2004; Tamborra et al., 2004; Selli et al., 2006; Lambropoulos and Roussis et al., 2007; Izquierdo Cañas et al., 2008; Nasi et al., 2008; RodriguezBencomo et al., 2008; Loscos et al., 2010; Antalick et al., 2010; Sáenz-Navajas et al., 2010). Quantification can be done with both detectors, while unequivocal identification only by MS.

On Agricultural institute of Slovenia we decided to introduce discontinuous liquid-liquid extraction method with dichloromethane, chosen as the most effective organic solvent for this type of extraction (Cabredo Pinillos et al., 2004). The extraction was performed with intention to determine 26 minor volatile compounds with possible sensorial effect in wines (Schneider et al., 1998; Selli et al., 2003; Selli et al., 2006; Lukić et al., 2008). Liquid-liquid extraction is actually the oldest but still the reference technique for the extraction of volatile compounds in wine (Ortega et al., 2002). 3-octanol and 4-nonanol were used as internal standards because of their high recovery (Cabredo Pinillos et al., 2004; Selli et al., 2006). By this procedure we achieved concentration factor 100 . To enable qualitative and quantitative evaluation at the same time, MS was used for detection. After introduction, method was validated. Method was finally applied to real wine samples (variety Zelen) deriving from an experiment, where two different winemaking procedures were confronted.

\section{MATERIALS AND METHODS}

\subsection{Materials}

Chemicals:

Dichloromethane (Sigma-Aldrich) and ethanol absolute (Merck) with HPLC grade were used like solvents in our experiment, together with ultrapure water from the Milli-Q system. Similarly only the volatile compounds (Merck, SigmaAldrich, Fluka, SAFC) with the highest available purity on market (minimum of $95 \%$ ) were used with the exception of 4 vinylphenol (SAFC) only sold like $10 \%$ solution.

\section{Preparation of solutions:}

Stock solutions in pure dichloromethane of individual volatiles were prepared in $50 \mathrm{ml}$ volumetric flasks with concentrations ranging from $1.8-2.5 \mathrm{~g} / \mathrm{L}$. From 26 stock solutions one mix solution of all 26 volatiles was prepared in $200 \mathrm{~mL}$ volumetric flask. All other solutions used to determine linearity, limits of detection and limits of quantification were prepared from this mix solution with proper dilutions.

Internal standards 3-octanol and 4-nonanol for those dichloromethane solutions were prepared in $100 \mathrm{~mL}$ volumetric flask with dissolving them in quantity of $1.1-1.2$ $\mathrm{g} / \mathrm{L}$ in dichloromethane. They were added using $0.05 \mathrm{~mL}$ Hamilton syringe to $10 \mathrm{~mL}$ of dichloromethane solutions and mixed before determination.

\section{Preparation of model wine solution:}

First a mix stock solution of all volatiles in $100 \%$ pure ethanol was prepared, with individual volatiles concentrations in range of $0.8-1.2 \mathrm{~g} / \mathrm{L}$. Stock solution was adequately diluted to model solution (mix) using $12 \%$ vol ethanol in water to concentrations similar to ones determined in wines in average, to $0.04-0.07 \mathrm{mg} / \mathrm{L}$, in $3000 \mathrm{ml}$ volumetric flask. The $\mathrm{pH}$ was then adjusted to $\mathrm{pH} 3.2$ with tartaric acid addition. Model wine solution was finally dispensed in twenty $125 \mathrm{~mL}$ flasks and they were stored in dark at $7{ }^{\circ} \mathrm{C}$ before extraction.

Internal standards 3-octanol and 4-nonanol used in our model wine solution were prepared in $100 \mathrm{~mL}$ volumetric flask with dissolving them in quantity of $0.04-0.06 \mathrm{~g}$ in ethanol absolute. They were added using $0.05 \mathrm{~mL}$ Hamilton syringe to model wine solution only during extraction process as described below. 


\subsection{Procedure}

Liquid-liquid extraction of volatile compounds:

$100 \mathrm{~mL}$ of model wine solution was transferred into $250 \mathrm{ml}$ Erlenmeyer flask and cooled to $0{ }^{\circ} \mathrm{C}$ in an ice bath under nitrogen. $29 \mu \mathrm{g}$ of 3-octanol and $23 \mu \mathrm{g}$ of 4-nonanol were added as internal standards using $0.05 \mathrm{~mL}$ Hamilton syringe from corresponding ethanol solutions. Dichloromethane (40 $\mathrm{mL}$ ) was added and the mixture was stirred at $350 \mathrm{~min}^{-1}$ for 20 minutes (Moio et al., 1995). Then the mixture was centrifuged at $5^{\circ} \mathrm{C}(\mathrm{RFC}=8500,10$ minutes $)$ and organic phase was recovered. The aqueous phase was re-extracted twice in the same way described above. Finally organic phases were combined and dried over sodium sulphate. They were concentrated to a final volume of $1 \mathrm{~mL}$ with Vigreaux distillation column and nitrogen gas flow prior to GC-MS analysis (Schneider et al., 1998).

The same procedure was used for the extraction of wine samples (Moio et al., 1995; Schneider et al., 1998; Selli et al., 2006).

\subsection{Determination}

Chromatographic conditions of GC (HP 6890)-MS (HP 5973) system:

\begin{tabular}{|c|c|}
\hline Liner & Agilent 5062-3587 \\
\hline Injector temperature & $200^{\circ} \mathrm{C}$ \\
\hline Injection type & Pulsed Splitless \\
\hline Precolumn & $2 \mathrm{~m} * 0.25 \mathrm{~mm}$ \\
\hline Column & Varian, CP-WAX 57CB, $50 \mathrm{~m} \times 0.25 \mathrm{~mm}$ ID \\
\hline Temperature gradient & $\begin{array}{l}40^{\circ} \mathrm{C} ; 12 \mathrm{~min} \\
5^{\circ} \mathrm{C} / \mathrm{min} \text {; from } 40{ }^{\circ} \mathrm{C} \text { to } 200^{\circ} \mathrm{C} \\
200^{\circ} \mathrm{C} ; 20 \mathrm{~min}\end{array}$ \\
\hline Ion source temperature & $230^{\circ} \mathrm{C}$ \\
\hline Auxiliary temperature & $200^{\circ} \mathrm{C}$ \\
\hline Detector temperature & $150^{\circ} \mathrm{C}$ \\
\hline Carrier gas & Helium 6.0 ; constant flow $1.0 \mathrm{ml} / \mathrm{min}$ \\
\hline Injection volume & $1 \mu \mathrm{l}$ \\
\hline Detection & $\begin{array}{l}\text { Selective Ion Monitoring }(\mathrm{T}, \mathrm{Q} 1, \mathrm{Q} 2, \mathrm{Q} 3) \text { : } \\
\text { 1,6-Heptadien-4-ol }(\mathbf{7 1}, 43) \\
\text { 1-Hexanol }(\mathbf{5 6}, 43,55,69) \\
\text { 2-Phenylethyl acetate }(\mathbf{1 0 4}, 43,91) \\
\text { 3-Octanol }(\mathbf{5 9}, 83,101) \\
\text { 4-Ethylguaiacol }(2-\text {-Methoxy-4-ethylphenol })(\mathbf{1 3 7}, 152) \\
\text { 4-Nonanol }(\mathbf{5 5}, 73,83,101) \\
\text { 4-Vinylguaiacol }(2-\text {-Methoxy-4-vinylphenol) }(\mathbf{1 5 0}, 135,107,77) \\
\text { 4-Vinylphenol }(\mathbf{1 2 0}, 91) \\
\text { Benzaldehyde }(\mathbf{7 7}, 105,106) \\
\text { Benzyl alcohol }(\mathbf{7 9}, 108,107) \\
\text { cis-3-hexen-1-ol }(\mathbf{6 7}, 41,82) \\
\text { Diethyl succinate }(\mathbf{1 0 1}, 129) \\
\text { Ethyl butyrate }(\text { Ethyl butanoate })(\mathbf{7 1}, 43,88) \\
\text { Ethyl cinnamate }(\mathbf{1 3 1}, 103,176) \\
\text { Ethyl decanoate }(\text { Ethyl caprate) }(\mathbf{8 8}, 101,155) \\
\text { Ethyl dodecanoate }(\text { Ethyl laurate) }(\mathbf{8 8}, 101) \\
\text { Ethyl hexadecanoate }(\text { Ethyl palamitate })(\mathbf{8 8}, 101) \\
\text { Ethyl hexanoate }(\mathbf{8 8}, 99) \\
\text { Ethyl lactate }(\mathbf{4 5}, 75) \\
\text { Ethyl octanoate }(\text { Ethyl caprylate) }(\mathbf{8 8}, 101,57) \\
\text { Geraniol }(\mathbf{6 9}, 93,123) \\
\text { Hexyl acetate }(\mathbf{4 3}, 56) \\
\text { Isoamyl acetate }(\mathbf{7 0}, 43,55) \\
\text { Nerol }(\mathbf{6 9}, 84,93) \\
\text { n-Hexaldehyde }(\text { Capronaldehyde) }(\mathbf{5 6}, 44,57) \\
\text { trans-2-hexen-1-ol }(\mathbf{5 5}, 69,83) \\
\beta \text {-Ionone }(\mathbf{1 7 7}, 43) \\
\gamma \text {-Butyrolactone }(\mathbf{4 2}, 56,86) \\
\end{array}$ \\
\hline
\end{tabular}


2.4 Aromatic compounds determination in wines from two winemaking procedures - preparation of wine samples

Healthy grapes of Zelen variety $(40 \mathrm{~kg}$ ) were manually harvested in 2008 at the ripeness stage corresponding to wines containing approximately $12 \%$ vol ethanol. Grapes were divided in two equal parts. First half of grapes $(\mathrm{Zc}=$ control without skin contact) was immediately destemmed, crushed and pressed up to $150 \mathrm{kPa}$ using a small water press (Lancman VS-A 55, Slovenia). The juice was sulphited with $30 \mathrm{mg} / \mathrm{L}$ of sulphur dioxide, left to settle at $6^{\circ} \mathrm{C}$ for 12 hours, racked and divided in three glass laboratory fermentor vessels with $1.6 \mathrm{~L}$ juice each. The vessels were heated to $17^{\circ} \mathrm{C}$, inoculated with $0.2 \mathrm{~g} / \mathrm{L}$ of dried Saccharomyces cerevisiae (CM, Lallemand), supplemented with $0.2 \mathrm{~g} / \mathrm{L}$ complex yeast nutrient (Fermaid E,
Lallemand) and fermented at $17^{\circ} \mathrm{C}$. After alcoholic fermentations (residual sugars $<2.5 \mathrm{~g} / \mathrm{L}$ ) and when most of the lees had settled, the wines were racked, $50 \mathrm{mg} / \mathrm{L}$ of sulphur dioxide was added and the wines were stored at $10^{\circ} \mathrm{C}$. The second half of grapes $(\mathrm{Zp}=$ freezing of the pomace) was destemmed and crushed. The pomace was equally divided in three plastic vessels, frozen overnight at $-20^{\circ} \mathrm{C}$, defrozen at $20^{\circ} \mathrm{C}$ and pressed up to $150 \mathrm{kPa}$. The juice from the individual plastic vessels was sulphited with $30 \mathrm{mg} / \mathrm{L}$ of sulphur dioxide, left to settle at $6^{\circ} \mathrm{C}$ for 12 hours, racked and poured in 3 glass laboratory fermentor vessels with $1.6 \mathrm{~L}$ juice each. The remaining procedure to obtain wines was the same as described previously. In this way, two different types of Zelen wines $(\mathrm{Zc}, \mathrm{Zp})$ in three repetitions were obtained.

\section{RESULTS AND DISCUSSION}

\subsection{Linearity, limits of detection, limits of quantification}

Linearity was verified by using the solutions of volatile compounds in dichloromethane (five repetitions for one concentration level, three to eight concentration levels for the calibration curve). Linearity and range were determined by linear regression, using the $\mathrm{F}$ test. Linear model is fit and remains linear over the range presented in Table 1. Limits of detection (LD) and limits of quantification (LOQ) were calculated from the calibration curve and are presented in Table 1.

Linearity was verified for wider range also and is presented in Table 2.

Concentration factor for wine samples was due to extraction 100, so realistic linearity range, LDs and LOQs are 100-times lower.

Table 1: Linearity, limits of detection, limits of quantification

\begin{tabular}{|l|c|c|c|c|}
\hline & linearity $\mathbf{( m g / L )}$ & $\mathbf{R}^{2}$ & $\begin{array}{c}\mathbf{L D} \\
\mathbf{( m g} / \mathbf{L})\end{array}$ & $\mathbf{L O Q} \mathbf{( m g / \mathbf { L } )}$ \\
\hline 1,6-Heptadien-4-ol & $0.12-3.35$ & 0.999 & 0.062 & 0.206 \\
\hline 1-Hexanol & $0.19-3.35$ & 0.999 & 0.067 & 0.224 \\
\hline 2-Phenylethyl acetate & $0.0168-3.35$ & 0.999 & 0.030 & 0.099 \\
\hline 4-Ethylguaiacol & $0.0058-3.35$ & 0.996 & 0.051 & 0.171 \\
\hline 4-Vinylguaiacol & $0.05-3.35$ & 0.998 & 0.062 & 0.208 \\
\hline 4-Vinylphenol & $0.05-3.35$ & 0.998 & 0.061 & 0.203 \\
\hline Benzaldehyde & $0.0111-1.67$ & 0.993 & 0.041 & 0.136 \\
\hline Benzylalcohol & $0.0116-1.67$ & 0.994 & 0.038 & 0.126 \\
\hline cis-3-Hexen-1-ol & $1.67-10.13$ & 0.996 & 0.352 & 1.174 \\
\hline Diethyl succinate & $0.022-3.35$ & 0.996 & 0.066 & 0.218 \\
\hline Ethyl butyrate & $0.0167-1.67$ & 0.999 & 0.016 & 0.052 \\
\hline Ethyl cinnamate & $0.05-3.35$ & 0.998 & 0.056 & 0.186 \\
\hline Ethyl decanoate & $0.05-1.67$ & 0.995 & 0.056 & 0.186 \\
\hline Ethyl dodecanoate & $0.01-3.35$ & 0.996 & 0.065 & 0.216 \\
\hline Ethyl hexadecanoate & $0.0139-1.67$ & 0.995 & 0.034 & 0.115 \\
\hline Ethyl hexanoate & $0.0092-3.35$ & 0.996 & 0.053 & 0.176 \\
\hline Ethyl lactate & $0.0099-1.67$ & 0.991 & 0.044 & 0.148 \\
\hline Ethyl octanoate & $0.1-9.51$ & 0.992 & 0.444 & 1.480 \\
\hline Geraniol & $0.011-3.35$ & 0.996 & 0.065 & 0.216 \\
\hline Hexyl acetate & $0.0092-1.67$ & 0.990 & 0.048 & 0.159 \\
\hline Isoamyl acetate & $0.05-3.35$ & 0.998 & 0.059 & 0.196 \\
\hline Nerol & $0.0058-3.35$ & 0.996 & 0.056 & 0.187 \\
\hline n-Hexaldehyde & $0.06-3.35$ & 0.983 & 0.175 & 0.582 \\
\hline trans-2-Hexen-1-ol & $0.05-3.35$ & 0.982 & 0.179 & 0.597 \\
\hline$\beta$-Ionone & $0.009-3.35$ & 0.995 & 0.074 & 0.248 \\
\hline$\gamma$-Butyrolactone & $0.024-3.35$ & 0.997 & 0.055 & 0.183 \\
\hline & & & & \\
\hline
\end{tabular}


Table 2: Linearity, wider range

\begin{tabular}{|l|c|c|}
\hline & linearity (mg/L) & $\mathbf{R}^{\mathbf{2}}$ \\
\hline 1,6-Heptadien-4-ol & $0.12-11.92$ & 0.961 \\
\hline 1-Hexanol & $0.19-19.4$ & 0.988 \\
\hline 2-Phenylethyl acetate & $0.0168-33.53$ & 0.995 \\
\hline 4-Ethylguaiacol & $0.0058-11.5$ & 0.987 \\
\hline 4-Vinylguaiacol & $0.05-9.36$ & 0.978 \\
\hline 4-Vinylphenol & $0.05-9.08$ & 0.977 \\
\hline Benzaldehyde & $0.0111-22.18$ & 0.994 \\
\hline Benzylalcohol & $0.0116-23.14$ & 0.994 \\
\hline cis-3-Hexen-1-ol & $1.67-10.13$ & 0.996 \\
\hline Diethyl succinate & $0.022-21.62$ & 0.993 \\
\hline Ethyl butyrate & $0.0167-33.45$ & 0.999 \\
\hline Ethyl cinnamate & $0.05-10.72$ & 0.983 \\
\hline Ethyl decanoate & $0.05-9.55$ & 0.977 \\
\hline Ethyl dodecanoate & $0.01-10.08$ & 0.978 \\
\hline Ethyl hexadecanoate & $0.0139-27.7$ & 0.973 \\
\hline Ethyl hexanoate & $0.0092-18.45$ & 0.989 \\
\hline Ethyl lactate & $0.0099-10.79$ & 0.986 \\
\hline Ethyl octanoate & $0.1-9.51$ & 0.992 \\
\hline Geraniol & $0.011-10.8$ & 0.985 \\
\hline Hexyl acetate & $0.0092-18.31$ & 0.989 \\
\hline Isoamyl acetate & $0.05-10.79$ & 0.983 \\
\hline Nerol & $0.0058-11.57$ & 0.988 \\
\hline n-Hexaldehyde & $0.06-11.31$ & 0.998 \\
\hline trans-2-Hexen-1-ol & $0.05-3.35$ & 0.982 \\
\hline$\beta$-Ionone & $0.009-9.32$ & 0.980 \\
\hline$\gamma$-Butyrolactone & $0.024-24.06$ & 0.994 \\
\hline
\end{tabular}

\subsection{Trueness}

Trueness was verified by checking the recoveries. Two parallel extracts of model wine solution were prepared each day for ten days and injected once respectively. The average of recoveries was calculated. The results are given in Table 3 .

\subsection{Precision}

For the determination of precision (ISO 5725), i.e. repeatability and reproducibility, extracts of model wine solution was analysed (the same as for recovery evaluation). Within the period of 10 days two parallel extracts were prepared each day. Each was injected once. Then standard deviation of repeatability of the level and standard deviation of reproducibility of the level were both calculated. The results are given in Table 4. 
Dejan BAVČAR, Helena BAŠA ČESNIK

Table 3: Recoveries for model wine solution

\begin{tabular}{|l|c|c|c|}
\hline & $\begin{array}{c}\text { conc. in model wine solution } \\
(\mathbf{m g} / \mathbf{L})\end{array}$ & $\begin{array}{c}\text { recovery } \\
\mathbf{( \% )}\end{array}$ & RSD (\%) \\
\hline 1,6-Heptadien-4-ol & 0.0435 & 84.4 & 3.1 \\
\hline 1-Hexanol & 0.0596 & 98.3 & 4.9 \\
\hline 2-Phenylethyl acetate & 0.0614 & 91.9 & 2.5 \\
\hline 3-Octanol & 0.2930 & 85.9 & 3.1 \\
\hline 4-Ethylguaiacol & 0.0543 & 92.3 & 4.2 \\
\hline 4-Nonanol & 0.2300 & 87.1 & 2.8 \\
\hline 4-Vinylguaiacol & 0.0692 & 95.0 & 8.9 \\
\hline 4-Vinylphenol & 0.0414 & 98.2 & 2.6 \\
\hline Benzaldehyde & 0.0713 & 95.0 & 3.0 \\
\hline Benzylalcohol & 0.0672 & 94.2 & 4.6 \\
\hline cis-3-Hexen-1-ol & 0.0566 & 84.0 & 3.0 \\
\hline Diethyl succinate & 0.0498 & 91.3 & 2.7 \\
\hline Ethyl butyrate & 0.0599 & 77.5 & 3.8 \\
\hline Ethyl cinnamate & 0.0697 & 95.1 & 2.9 \\
\hline Ethyl decanoate & 0.0500 & 81.9 & 15.2 \\
\hline Ethyl dodecanoate & 0.0624 & 67.8 & 8.9 \\
\hline Ethyl hexadecanoate & 0.0524 & 27.9 & 9.5 \\
\hline Ethyl hexanoate & 0.0588 & 76.7 & 3.4 \\
\hline Ethyl lactate & 0.0709 & 79.4 & 3.5 \\
\hline Ethyl octanoate & 0.0573 & 71.1 & 7.4 \\
\hline Geraniol & 0.0495 & 105.7 & 3.0 \\
\hline Hexyl acetate & 0.0593 & 80.6 & 4.0 \\
\hline Isoamyl acetate & 0.0604 & 78.0 & 3.8 \\
\hline Nerol & 0.0540 & 96.5 & 2.4 \\
\hline n-Hexaldehyde & 0.0433 & 82.0 & 3.3 \\
\hline trans-2-Hexen-1-ol & 0.0548 & 102.4 & 8.2 \\
\hline$\beta$-Ionone & 0.0564 & 89.2 & 2.6 \\
\hline$\gamma$-Butyrolactone & 0.0609 & 3.1 & 3.1 \\
\hline
\end{tabular}

Table 4: Standard deviation of repeatability and reproducibility of the method, in $\mathrm{mg} / \mathrm{L}$

\begin{tabular}{|c|c|c|c|c|}
\hline & $\begin{array}{c}\text { conc. in model wine } \\
\text { solution }(\mathrm{mg} / \mathrm{L})\end{array}$ & $\begin{array}{c}\text { means of the levels } \\
(\mathrm{mg} / \mathrm{L})\end{array}$ & $\begin{array}{c}\text { standard deviation of } \\
\text { repeatability (sr) }\end{array}$ & $\begin{array}{l}\text { standard deviation of } \\
\text { reproducibility }(s R)\end{array}$ \\
\hline 1,6-Heptadien-4-ol & 0.0435 & 0.0367 & 0.0007 & 0.0011 \\
\hline 1-Hexanol & 0.0596 & 0.0583 & 0.0011 & 0.0029 \\
\hline 2-Phenylethyl acetate & 0.0614 & 0.0563 & 0.0012 & 0.0014 \\
\hline 4-Ethylguaiacol & 0.0543 & 0.0501 & 0.0020 & 0.0024 \\
\hline 4-Vinylguaiacol & 0.0692 & 0.0659 & 0.0055 & 0.0056 \\
\hline 4-Vinylphenol & 0.0414 & 0.0406 & 0.0009 & 0.0010 \\
\hline Benzaldehyde & 0.0713 & 0.0676 & 0.0013 & 0.0021 \\
\hline Benzylalcohol & 0.0672 & 0.0632 & 0.0014 & 0.0028 \\
\hline cis-3-Hexen-1-ol & 0.0566 & 0.0475 & 0.0010 & 0.0014 \\
\hline Diethyl succinate & 0.0498 & 0.0455 & 0.0012 & 0.0012 \\
\hline Ethyl butyrate & 0.0599 & 0.0463 & 0.0010 & 0.0018 \\
\hline Ethyl cinnamate & 0.0697 & 0.0662 & 0.0014 & 0.0019 \\
\hline Ethyl decanoate & 0.0500 & 0.0404 & 0.0010 & 0.0064 \\
\hline Ethyl dodecanoate & 0.0624 & 0.0419 & 0.0008 & 0.0039 \\
\hline Ethyl hexadecanoate & 0.0524 & 0.0147 & 0.0012 & 0.0014 \\
\hline Ethyl hexanoate & 0.0588 & 0.0449 & 0.0010 & 0.0016 \\
\hline Ethyl lactate & 0.0709 & 0.0562 & 0.0015 & 0.0020 \\
\hline Ethyl octanoate & 0.0573 & 0.0405 & 0.0009 & 0.0031 \\
\hline Geraniol & 0.0495 & 0.0524 & 0.0014 & 0.0015 \\
\hline Hexyl acetate & 0.0593 & 0.0476 & 0.0011 & 0.0019 \\
\hline Isoamyl acetate & 0.0604 & 0.0470 & 0.0012 & 0.0017 \\
\hline Nerol & 0.0540 & 0.0521 & 0.0012 & 0.0012 \\
\hline n-Hexaldehyde & 0.0433 & 0.0354 & 0.0007 & 0.0012 \\
\hline trans-2-Hexen-1-ol & 0.0548 & 0.0559 & 0.0044 & 0.0050 \\
\hline$\beta$-Ionone & 0.0564 & 0.0503 & 0.0010 & 0.0013 \\
\hline$\gamma$-Butyrolactone & 0.0609 & 0.0536 & 0.0012 & 0.0016 \\
\hline
\end{tabular}




\subsection{Uncertainty of repeatability and uncertainty of reproducibility}

Uncertainty of repeatability and uncertainty of reproducibility were calculated by multiplying standard deviation of repeatability and standard deviation of reproducibility by Student's $t$ factor for 9 degrees of freedom and $95 \%$ confidence level $\left(t_{95 ; 9}=2.262\right)$.

$U_{r}=t_{95 ; 9} \times s_{r} ; U_{R}=t_{95 ; 9} \times s_{R}$

The results are presented in Table 5.

Table 5: Uncertainty of repeatability and reproducibility of the method, in $\mathrm{mg} / \mathrm{L}$

\begin{tabular}{|l|c|c|c|}
\hline & $\begin{array}{c}\text { conc. in model wine solution } \\
(\mathbf{m g} / \mathbf{L})\end{array}$ & $\begin{array}{c}\text { uncertainty of repeatability } \\
\left(\boldsymbol{U}_{\boldsymbol{r}}\right)\end{array}$ & $\begin{array}{c}\text { uncertainty of reproducibility } \\
\left(\boldsymbol{U}_{\boldsymbol{R}}\right)\end{array}$ \\
\hline 1,6-Heptadien-4-ol & 0.0435 & 0.0016 & 0.0026 \\
\hline 1-Hexanol & 0.0596 & 0.0025 & 0.0066 \\
\hline $\begin{array}{l}\text { 2-Phenylethyl } \\
\text { acetate }\end{array}$ & 0.0614 & 0.0026 & 0.0031 \\
\hline 4-Ethylguaiacol & 0.0543 & 0.0046 & 0.0055 \\
\hline 4-Vinylguaiacol & 0.0692 & 0.0124 & 0.0127 \\
\hline 4-Vinylphenol & 0.0414 & 0.0021 & 0.0023 \\
\hline Benzaldehyde & 0.0713 & 0.0029 & 0.0047 \\
\hline Benzylalcohol & 0.0672 & 0.0031 & 0.0064 \\
\hline cis-3-Hexen-1-ol & 0.0566 & 0.0022 & 0.0032 \\
\hline Diethyl succinate & 0.0498 & 0.0026 & 0.0027 \\
\hline Ethyl butyrate & 0.0599 & 0.0022 & 0.0040 \\
\hline Ethyl cinnamate & 0.0697 & 0.0031 & 0.0042 \\
\hline Ethyl decanoate & 0.0500 & 0.0023 & 0.0144 \\
\hline Ethyl dodecanoate & 0.0624 & 0.0017 & 0.0088 \\
\hline Ethyl \\
hexadecanoate & 0.0524 & 0.0027 & 0.0031 \\
\hline Ethyl hexanoate & 0.0588 & 0.0023 & 0.0036 \\
\hline Ethyl lactate & 0.0709 & 0.0034 & 0.0044 \\
\hline Ethyl octanoate & 0.0573 & 0.0021 & 0.0071 \\
\hline Geraniol & 0.0495 & 0.0031 & 0.0034 \\
\hline Hexyl acetate & 0.0593 & 0.0025 & 0.0044 \\
\hline Isoamyl acetate & 0.0604 & 0.0028 & 0.0040 \\
\hline Nerol & 0.0540 & 0.0027 & 0.0028 \\
\hline n-Hexaldehyde & 0.0433 & 0.0016 & 0.0026 \\
\hline trans-2-Hexen-1-ol & 0.0548 & 0.0100 & 0.0113 \\
\hline$\beta$-Ionone & 0.0564 & 0.0022 & 0.0029 \\
\hline$\gamma$-Butyrolactone & 0.0609 & 0.0028 & 0.0036 \\
\hline
\end{tabular}

\subsection{Aromatic compounds determination in wines from two winemaking procedures}

To determine volatile compound in real wine samples, the method proposed in this article was applied and results are presented in Table 6. Results are in correlation with previously observed aromatics content in wines and differences due to two winemaking procedures are comparable to other skin contact procedures (Moio et al., 1995; Ortega et al., 2002; Selli et al., 2003; Selli et al., 2006; Rodriguez-Bencomo et al., 2008). 
Table 6 : Concentrations of individual aromatic compounds in the Zelen wines produced by two different procedures ( $\mathrm{Zc}$ - control without skin contact, $\mathrm{Zp}$ - freezing of pomace), in $\mu \mathrm{g} / \mathrm{L}$.

\begin{tabular}{|c|c|c|}
\hline & Zc wine & Zp wine \\
\hline 1,6-Heptadien-4-ol & $17 \pm 1^{\mathrm{b}}$ & $12 \pm 1^{\mathrm{a}}$ \\
\hline 1-Hexanol & $1349 \pm 67^{b}$ & $1118 \pm 18^{\mathrm{a}}$ \\
\hline 2-Phenylethyl acetate & $466 \pm 49^{b}$ & $253 \pm 12^{\mathrm{a}}$ \\
\hline 4-Ethylguaiacol & - & - \\
\hline 4-Vinylguaiacol & $516 \pm 8^{\mathrm{a}}$ & $908 \pm 53^{\mathrm{b}}$ \\
\hline 4-Vinylphenol & $117 \pm 5^{\mathrm{a}}$ & $354 \pm 23^{\mathrm{b}}$ \\
\hline Benzaldehyde & $2 \pm 0^{\mathrm{a}}$ & $9 \pm 2^{b}$ \\
\hline Benzylalcohol & $20 \pm 3^{\mathrm{a}}$ & $90 \pm 9^{b}$ \\
\hline cis-3-Hexen-1-ol & $21 \pm 1^{\mathrm{b}}$ & $18 \pm 0^{\mathrm{a}}$ \\
\hline Diethyl succinate & $112 \pm 16^{\mathrm{a}}$ & $129 \pm 8^{\mathrm{a}}$ \\
\hline Ethyl butyrate & $366 \pm 10^{\mathrm{b}}$ & $217 \pm 11^{\mathrm{a}}$ \\
\hline Ethyl cinnamate & - & - \\
\hline Ethyl decanoate & $593 \pm 6^{\mathrm{b}}$ & $443 \pm 9^{a}$ \\
\hline Ethyl dodecanoate* & $37 \pm 2^{\mathrm{a}}$ & $38 \pm 4^{\mathrm{a}}$ \\
\hline Ethyl hexadecanoate* & $6 \pm 1^{\mathrm{a}}$ & $8 \pm 1^{\mathrm{a}}$ \\
\hline Ethyl hexanoate & $570 \pm 16^{\mathrm{b}}$ & $409 \pm 0^{\mathrm{a}}$ \\
\hline Ethyl lactate & $4276 \pm 475^{a}$ & $6350 \pm 328^{b}$ \\
\hline Ethyl octanoate & $1239 \pm 56^{\mathrm{b}}$ & $933 \pm 25^{\mathrm{a}}$ \\
\hline Geraniol & - & - \\
\hline Hexyl acetate & $275 \pm 40^{\mathrm{b}}$ & $46 \pm 5^{\mathrm{a}}$ \\
\hline Isoamyl acetate & $3260 \pm 423^{b}$ & $1291 \pm 97^{\mathrm{a}}$ \\
\hline Nerol & - & - \\
\hline n-Hexaldehyde & $4 \pm 1^{b}$ & $-{ }^{a}$ \\
\hline trans-2-Hexen-1-ol & - & - \\
\hline$\beta$-Ionone & - & - \\
\hline$\gamma$-Butyrolactone & $2501 \pm 60^{\mathrm{a}}$ & $2569 \pm 62^{\mathrm{a}}$ \\
\hline
\end{tabular}

Values are the mean value \pm error at $95 \%$ confidence level $(n=3)$.

Significant differences between procedures are indicated $\mathrm{a}, \mathrm{b}$ at $\mathrm{p} \leq 0.05$.

- = not detected

* = volatile compounds with low recoveries

\section{CONCLUSIONS}

According to the validation, the method is suitable for the determination of at least 24 volatile compounds in wine (the ones with recoveries $>70 \%$ ). The system is linear with $\mathrm{R}^{2}$ higher than 0.96 . Limits of detection range from $0.16 \mu \mathrm{g} / \mathrm{L}$ for ethyl butyrate to $4.44 \mu \mathrm{g} / \mathrm{L}$ for ethyl octanoate. Limits of quantitative determination range from $0.52 \mu \mathrm{g} / \mathrm{L}$ for ethyl butyrate to $14.8 \mu \mathrm{g} / \mathrm{L}$ for ethyl octanoate. Recoveries range from $71.1 \%$ (ethyl octanoate) to $105.7 \%$ (geraniol), except for ethyl dodecanoate $(67.8 \%)$ and ethyl hexadecanoate $(27.9 \%)$. Uncertainty of reproducibility ranges from $5.0 \%$ for 2 phenylethyl acetate to $28.9 \%$ for ethyl decanoate. A practical application was checked and presented for Zelen wines from two different winemaking procedures.

\section{ACKNOWLEGEMENTS}

The authors thank those who contributed to the work: Mr. Tomaž Sket and co-workers at the Central Laboratories of the Agricultural Institute of Slovenia. 


\section{REFERENCES}

Antalick, G., Perello, M.C. and de Revel G. 2010. Development, validation and application of a specific method for the quantitative determination of wine esters by headspace-solid-phase microextraction-gas chromatography-mass spectrometry. Food Chem., 121, 1236-1245.

Cabredo Pinillos, S., Cedron Fernandez, T. and Saent Barrio, C. 2004. Comparison of different extraction methods applied to volatile compounds in wine samples previous to the determination by gas chromatography. Anal. Lett., $37,3063-3084$.

ISO 5725: Accuracy (trueness and precision) of measurement methods and results - Part2: Basic method for the determination of repeatability and reproducibility of a standard measurement method, 1994, pp. 1-42.

Izquierdo Cañas, P.M., García Romero, E., Gómez Alonso, S. and Palop Herreros, M.L.L. 2008. Changes in the aromatic composition of Tempranillo wines during spontaneous malolactic fermentation. J. Food Compos. Anal., 21, 724-730.

Lambropoulos, I. and Roussis, I. G. 2007. Inhibition of the decrease of volatile esters and terpenes during storage of a white wine and a model wine medium by caffeic acid and gallic acid. Food Res. Int., 40, 176-181.

Loscos, N., Hernández-Orte, P., Cacho, J. and Ferreira, V. 2010. Evolution of the aroma composition of wines supplemented with grape flavour precursors from different varietals during accelerated wine ageing. Food Chem., 120, 205-216.

Lukić, I., Plavša, T., Sladonija., B., Radeka, S. and Perusic, D. 2008. Aroma compounds as markers of wine quality in the case of Malvazija Istarska young wine. J. Food Quality, 31, 717- 735.

Moio, L., Chambellant, E., Lasschaeve, I., Issanchau, S., Schlich, P. and Etievant, P. X. 1995. Production of representative wine extracts for chemical and olfactory analyses. It. J. Food Sci., 7, $265-278$.

Nasi, A., Ferranti, P., Amato, S. and Chianese, L. 2008. Identification of free and bound volatile compounds as typicalness and authenticity markers of non-aromatic grapes and wines through a combined use of mass spectrometric techniques. Food Chem., 110, 762-768.
Ortega Heras, M., Gonzales san Jose, M. L. and Belatran, S. 2002. Aroma composition of wine studied by different extraction methods. Anal. Chim. Acta, 458, 85- 93.

Peinado, R.A., Moreno, J.A., Munoz, D., Medina, M. and Moreno, J. 2004. Gas chromatographic quantification of major volatile compounds and polyols in wine by direct injection. J. Agr. Food Chem., 52, 6389 - 6393.

Pérez-Coello, M.S., González-Viñas, M.A., García-Romero, E., Díaz-Maroto, M.C. and Cabezudo, M.D. 2003. Influence of storage temperature on the volatile compounds of young white wines. Food Control, 14, 301-306.

Rodriguez-Bencomo, J.J., Méndez-Siverio, J.J., Pérez-Trujillo, J.P. and Cacho, J. 2008. Effect of skin conact on bound aroma and free volatiles of Listán blanco wine. Food Chem., 110, 214-225.

Sáenz-Navajas, M.-P., Campo, E., Fernández-Zurbano, P. and Valentin, D. 2010. An assessment of the effects of wine volatiles on the perception of taste and astringency in wine. Food Chem., 121, 1139-1149.

Schneider, R., Baumes, R. L., Bayonove, C.L. and Razungles, A. 1998, Volatile compounds involved in the aroma of sweet fortified wines (Vins Doux Naturels) from Grenache Noir. J. Agr. Food Chem., 46, 3230 - 3237.

Selli, S., Cabaroglu, T., Canbas, A., Erten, H. and Nurgel, C. 2003. Effect of skin contact on the aroma composition of the musts of Vitisvinifera L. cv. Muscat of Bornova and Narince grown in Turkey. Food Chem., 81, 341-347.

Selli, S., Cabaroglu, T., Canbas, A., Erten, H., Nurgel, C., Lepoutre, J.P. and Gunata, Z. 2004. Volatile composition of red wine from cv. Kalecik Karasu grown in central Anatolia. Food Chem., 85, 207-213.

Selli, S., Canbas, A., Cabaroglu, T., Erten, H. and Gunata, Z. 2006. Aroma components of cv. Muscat of Bornova wines and influence of skin contact treatment. Food Chem., 94, 319-326.

Tamborra, P., Martino, N. and Esti, M. 2004. Laboratory tests on glycosidase preparations in wine. Anal. Chim. Acta, 513, 299-303. 